\title{
Data Packet Security in MANET and VANET
}

\author{
Zalte S.S, Patil P.N ${ }^{2}$, Deshmukh S.N. ${ }^{2}$ and Kamat R. K. ${ }^{2}$ \\ ${ }^{1}$ Computer Science, Shivaji University, Kolhapur, India \\ ${ }^{2}$ Computer Science,Vishwakarma College, Pune, India
}

\section{ABSTRACT}

Day by Day security turns into a significant worry in delicate or confidential information transmission in Mobile Ad-hoc network (MANET) or Vehicular Ad-hoc Network (VANET). In this type of network, topology of nodes is unpredictable because of its changing nature. Without permission, participation and departure of nodes results in lack of trust relationship between nodes. In such conditions, there is no assurance that way between two nodes would be secure or free of malicious nodes. These nodes may impose variety of attacks like capturing sensitive information or modifying it for their self benefit or bad intention. In this paper we present hybrid cryptographic to upgrade protection to data packets, which is amalgamation of symmetric cryptography, asymmetric cryptography and hashing to provide shield to data packets.

\section{KEY WORDS: ASYMMETRIC CRYPTOGRAPHY, MANET, MALICIOUS NODES, SYMMETRIC CRYPTOGRAPHY, VANET.}

\section{INTRODUCTION}

Generally, the data is extremely confident about the business or domestic environment. So information security assumes imperative part in these conditions. There are numerous cryptographic procedures accessible for information security. Every method works in its remarkable manner and has its own aces and cons. During routing of data packets, it is vital to give security to path just as data packets. This paper presents hybrid cryptography method to upgrade information security in routing protocol. It attempts to give shield to data packets. All data packets are concealed utilizing prominent security cryptographic algorithms. The proposed data packet security accomplishes all security primitives, for example, Authentication, Confidentiality, Integrity and non-repudiation and so on.

\section{ARTICLE INFORMATION}

*Corresponding author email: sheetal.zaltegaikwad@gmail.com Received 10th Oct 2020 Accepted after revision 27th Dec 2020 Print ISSN: 0974-6455 Online ISSN: 2321-4007 CODEN: BBRCBA

Thomson Reuters ISI Web of Science Clarivate Analytics USA and Crossref Indexed Journal

\section{Clarivate $\begin{gathered}\text { Cnalytics } \\ \text { Crossef }\end{gathered}$}

NAAS Journal Score 2020 (4.31)

A Society of Science and Nature Publication,

Bhopal India 2020. All rights reserved.

Online Contents Available at: http//www.bbrc.in/

Doi: http://dx.doi.org/10.21786/bbrc/13.15/47
VANET is one of type of MANET as shown in figure 1. In vehicular Ad-hoc Network (VANET), vehicles are used as router to communicate and forward information to vehicles and Road Side Unit (RSU) (Sharma,2019). Without any centralized authority, instant infrastructure is built in variety of applications like weather prediction, it is used to collect information about highway railway intersection, toll collection, nearest restaurant, petrol station, availability about parking area, improve traffic efficiency etc.

Figure 1: Architecture of MANET and VANET

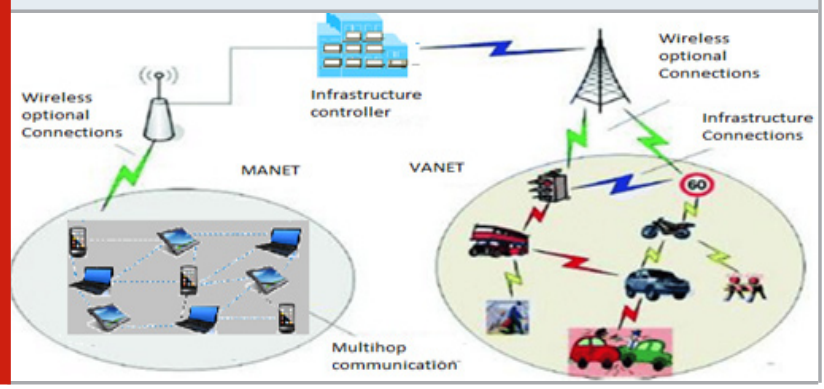


Some sensitive areas like military applications, war field of ad hoc networks where security is topmost priority that requires the most highly secure routing of data messages. In this case the applications that run on top of the network are of critical importance; therefore the underlying routing process should provide a high level of protection, while possibly having less strict performance requirements.

Weakness in the security system is called vulnerability. MANET is more vulnerable than a wired network. Mobile Ad-hoc Network has very constrained network compared to the traditional computer network. It has different characteristics like imprudent structure, dynamic topology, and promiscuous behavior. Resourceconstrained environment (memory, energy or battery power, CPU capacity and bandwidth) poses new challenges about security in MANET. (Ramayti, 2014)

\section{Obstacles in MANET and VANET}

- Imprudent structure: - MANET has lack of central authority. System allows anyone to access data without verifying authorization.

- Scalability: -Nodes in MANET are mobile which results in scalability of topology all the time. While concerning security, scalability is a major issue. So security mechanism must be versatile to handle a large network as well as small ones.

- Promiscuous mode: - When sender node transmits the packets, its neighbor nodes can overhear the transmission. If among them one node is malicious, it can corrupt the whole transmission.

- Resource constrained:-It is resource constrained in terms of data memory space, power supply etc. If power supply is limited, then nodes may behave selfishly.

- Asymmetric links: - Most of the wired networks rely on the symmetric links which are always fixed. But this does not happen in the case with VANET as the nodes are highly mobile and constantly changing their position with high speed in the network. For example, consider a MANET ( Mobile Ad-hoc Network )where node B sends a signal to node A but this does not tell anything about the quality of the connection in the reverse direction.( Liang, W,2015)

- Dynamic topology: - Due to mobility of node, relationships between the nodes changes from time to time. Establishing of trust becomes very difficult. When nodes are compromised, trust is disturbed. Adaptive security mechanism is a solution for this dynamic behavior. It is very difficult to detect malicious attacks because of lack of centralized management and highly dynamic environment. (Gorantala, K., 2006; Goyal, P)

- Ease of snooping data: - While transmitting data in the MANET, other devices acknowledges about transmission of data. So it is easy for intruder to snoop data or information which is transferred over the network.

- Routing: - Various demands like uncasing, multicasting and geo-casting from the network lead to performance degradation in MANET because network topology is unpredictable and dynamic.

- Packet loss: -In MANET packet loss occurs due to transmission error (limited transmission range) and frequently pathway breakages. There is no restriction on the nodes in network participation, so malicious nodes can capture or drop packets for its personnel benefit (Raza, N., 2016).

- Lack of a Clear Line of Defense and Secure Communication: - MANETs do not have a clear line of defense; attacks can come from all directions. However, cryptography and authentication are difficult tasks in a mobile wireless environment since they consume significant resources.

\section{Cryptographic Algorithm}

A. Advance Encryption Standard (AES): The core characteristics of AES are, it provides elevated security with minimum overhead, It has small key size, consumes less energy, processing speed is high, robust,

simple in design . These characteristics are valuable in resource constrained environments like in VANET. In AES block and key sizes are vary. If the key size is 128 bits-10 rounds are required. If it is 192 bit-12 rounds required. 128 bit key is commonly used everywhere.

B. Dual RSA: In proposed method dual RSA is used to reduce memory consumption and achieve elevated security. It stores, public and private exponent only once, not twice for two distinct instances of RSA (Hung-Min Sun, 2007). In RSA two keys are generated public key (e, n) and private key (d, n). Now for enforcing high security, we again perform key generation operation by choosing private key $d$ and $n$. Then these newly generated public and private key pairs are used in digital signature. Dual RSA used for authentication and non-repudiation.

C. Secure Hash Algorithm2 (SHA2): Hashing is cryptographic function used to map data of any size into fixed size digest. It is used to achieve integrity and digital signature. In this proposed method, SHA-256 algorithm used. Hashing is popular because of It is irreversibility.

Proposed Method: The proposed method includes hybrid architecture, which is a combo of symmetric and asymmetric cryptography. The Symmetric Key Cryptographic Technique (Advanced Encryption Standard) and hashing technique (SHA2) are used to achieve Confidentiality and Integrity, respectively. In asymmetric Key Cryptography technique Authentication is achieved by Dual RSA. The digital signature is used to achieve non-repudiation. Our goal is to guarantee confidentiality, authentication, packet integrity and non-repudiation during packet transmission.

\section{The source side}

1. The given plain text can be encrypted with the help of Advanced Encryption Standard, AES and the derived cipher text can be transferred to the destination.

2. At the same time value is calculated through SHA2 for the same encrypted plain text, which is also 
converted into the cipher text by AES.

3. This encrypted text and hash value encrypted text send to the destination.

4. This Hash value of plain text has been encrypted with Dual RSA and the encrypted message of this hash value is also sent to destination.

Source Side

$$
\begin{aligned}
& =\int_{i=0}^{\text {PacketsN }} \text { forward }(\operatorname{aes}(i)+\operatorname{hash}(\operatorname{aes}(i)))+\text { forward }(\text { hash }(i) \\
& +\operatorname{drsa}(\operatorname{hash}(i)))
\end{aligned}
$$

5. Intermediate nodes simply forward the packets to next hop.

$$
\begin{gathered}
\text { Intermidiate Node }=\int_{i=0}^{\text {PathN }-1} i==\operatorname{Secure} \rightarrow \operatorname{Packet}(i) \\
\rightarrow \operatorname{Packet}(\operatorname{Next} \operatorname{Hop}(i+1))
\end{gathered}
$$

\section{The destination side}

1. The hash value of encrypted plain text by AES, is calculated and it is compare with hash digest of computed data.

$$
\begin{aligned}
& \text { Receiving Nodes(aes) } \\
& =\int_{i=0}^{\text {PacketsN }} \operatorname{hash}(\operatorname{aes}(i))= \\
& =(\operatorname{hash}(i))\left\{\begin{array}{c}
\text { match }=\text { Accept } \text { Packet aes }[i]=\text { true } \\
\text { Not match }=\text { Reject } \text { Packet }
\end{array}\right.
\end{aligned}
$$

2. At same time, the received encrypted hash value is decrypted by dual RSA to get original hash value.

3. This decrypted hash value is compared with the original Hash value $\mathrm{H} 1$ which is calculated initially.

$$
\begin{aligned}
& \text { Receiving Nodes }(\operatorname{drsa})= \\
& \quad \int_{i=0}^{\text {PacketsN } \operatorname{decrypt}(\operatorname{drsa}(\text { hash }(i))==(\text { hash }(i))}\left\{\begin{array}{c}
\text { match }=\text { Accept Packet } r s a M[i]=\text { true } \\
\text { Not match }=\text { Reject Packet }
\end{array}\right.
\end{aligned}
$$

4. If both side's hash values match, then message is authenticated and it is not altered. So at receiver's side message is accepted.

5. Otherwise message is dropped or rejected.

$$
\text { Receiving Nodes }=\int_{i=0}^{\text {PacketsN }} \operatorname{aes} M[i]==\operatorname{rsaM}[i]\left\{\begin{array}{l}
\text { match }=\operatorname{decrypt}(\operatorname{aes}(i)) \\
\text { Not } \text { match }=\operatorname{Reject} \text { Packet }
\end{array}\right.
$$

While considering different kind of attacks we should also look for interceptor. When data is sent in encrypted format interceptor determines plain text from encrypted data very easily. Most of the time the interceptor derives both encrypted message and also hash value for that particular message. Once it has both he easily derives the plain text from encrypted text. But in this proposed work if it gets hash value, it cannot derive plain text because we have used dual RSA to encrypt hash value and original data is encrypted by AES algorithm which is highly secure.

\section{Security Analysis}

Unauthorized Nodes: - Only legitimate nodes having private key are permitted to participate in network. Secrete keys are distributed prior to routing starts. Without signature generated packet is dropped by the receiver.

Modification: - For non-legitimate node or other nodes, it is very difficult to modify the message contents because messages are signed by the sender.

Fabrication:- Only those nodes having the private key can able to fabricate messages.

Impersonation:- lack of authentication leads fake nodes can become legitimate nodes and able to observe data traffic. These types of users also prohibited from sending fake routing messages. (Sharma, M,2020)

\section{CONCLUSION}

Security of data transmission becomes hot topic in digital world. There are so many cryptographic techniques are available to provide elevated security to routing protocols. These techniques are required to provide data security and users authentication. The proposed method has been designed for better security using a combo of different cryptographic techniques. In the proposed hybrid architecture of cryptographic techniques such as Advanced Encryption Standard, and SHA2 are used to achieve both the Confidentiality and Integrity. To achieve authentication, we used asymmetric Key algorithm, Dual RSA. Digital signature used to achieve non-repudiation.

\section{REFERENCES}

Gorantala, K., 2006. Routing protocols in mobile ad-hoc networks. A Master'thesis in computer science, pp-136.

Goyal, P., Batra, S. and Singh, A., 2010. A literature review of security attack in mobile ad-hoc networks. International Journal of Computer Applications, 9(12), pp.11-15.

Hung-Min Sun, Mu-En Wu, Wei-Chi Ting, and M. J. Hinek, Aug. 2007. Dual RSA and its security analysis. Journal IEEE Transactions on Information Theory, vol. 53, no. 8, , pp. 2922-2933. https://tools.cisco. com/security/center/resources/next_generation_ cryptography

Liang, W., Li, Z., Zhang, H., Wang, S., \& Bie, R. (2015). Vehicular ad hoc networks: architectures, research issues, methodologies, challenges, and trends. International Journal of Distributed Sensor Networks, 11(8), 745303.

Raza, N., Aftab, M.U., Akbar, M.Q., Ashraf, O. and Irfan, M., 2016. Mobile ad-hoc networks applications and its challenges. Communications and Network, 8(3), pp.131- 
136.

Rmayti, M., Begriche, Y., Khatoun, R., Khoukhi, L. and Gaiti, D., 2014, September. Denial of Service (DoS) attacks detection in MANETs through statistical models. In 2014 Global Information Infrastructure and Networking Symposium (GIIS) (pp. 1-3). IEEE.
Sharma, M., \&t Rashid, M. (2020). Security Attacks In MANET-A Comprehensive Study. Available at SSRN 3565860.

Sharma, S. 2019. Vehicular ad-hoc network: An overview. In 2019 International Conference on Computing, Communication, and Intelligent Systems (ICCCIS) (pp. 131-134). IEEE. 